\title{
Community-acquired methicillin-resistant Staphylococcus epidermidis pyelonephritis in a child: a case report
}

\author{
Hiroaki Kanai", Hiroki Sato and Yoshichika Takei
}

\begin{abstract}
Introduction: Staphylococcus epidermidis is currently the most frequent pathogen of opportunistic and nosocomial infections worldwide. Most cases of Staphylococcus epidermidis infections are associated with indwelling medical devices and/or immunocompromised conditions. Community-acquired urinary tract infections are rare, particularly among pediatric populations, and clinicians often do not consider Staphylococcus epidermidis as a uropathogen.

Case presentation: A previously healthy Japanese boy developed pyelonephritis caused by Enterococcus faecalis at 10 months of age. Subsequently, he was diagnosed with severe bilateral vesicoureteral reflux (right side grade $\mathrm{V}$, left side grade III), and was administered trimethoprim/sulfamethoxazole as the prophylaxis. At 18 months of age, he presented with fever. Gram staining of urine obtained through catheterization revealed gram-positive cocci. We suspected pyelonephritis caused by enterococci, and administered oral fluoroquinolone empirically. The fever promptly resolved, and eventually, methicillin-resistant Staphylococcus epidermidis was detected at significant levels in the urine. Thus, our final diagnosis was pyelonephritis caused by community-acquired methicillin-resistant Staphylococcus epidermidis.

Conclusions: Our case indicated that even immunocompetent children without a urinary catheter can develop Staphylococcus epidermidis pyelonephritis. Staphylococcus epidermidis can be underdiagnosed or misdiagnosed as sample contamination in community-acquired urinary tract infections. Therefore, when Gram staining of appropriately obtained urine samples reveals gram-positive cocci, clinicians should take into consideration not only the possibility of enterococci but also staphylococci, including Staphylococcus epidermidis, particularly in children with urinary abnormalities and/or those receiving continuous antibiotic prophylaxis.
\end{abstract}

Keywords: Methicillin-resistant Staphylococcus epidermidis, Pyelonephritis, Vesicoureteral reflux, Antibiotic prophylaxis

\section{Introduction}

Staphylococcus epidermidis (S. epidermidis) is one of the most common pathogens of nosocomial and opportunistic infections, and is generally associated with infections caused by indwelling foreign devices, including central vascular catheters, cerebrospinal fluid shunts or prosthetic cardiac valves, as well as by immunocompromised conditions [1,2]. S. epidermidis is also the most common pathogen of nosocomial bacteremia in children, particularly in neonatal intensive care units, and is a common pathogen of healthcare-associated bacteremia in patients

\footnotetext{
* Correspondence: hkanai@kind.ocn.ne.jp

Department of Pediatrics, Suwa Central Hospital, Tamagawa 4300, Chino-city, Nagano 391-8503, Japan
}

of all age groups [2]. Furthermore, prosthetic valve endocarditis, central venous catheter infections and cerebrospinal fluid shunt meningitis have also been documented to be caused by $S$. epidermidis [1]. However, community-acquired infections caused by $S$. epidermidis in immunocompetent children are rarely reported, and the etiology is unclear.

S. epidermidis was not reported as a uropathogen in recent studies of urinary tract infections (UTIs) [3-5]. Although information is limited and S. epidermidis infection may often be underdiagnosed or misdiagnosed as sample contamination, it appears evident that, overall, the prevalence of S. epidermidis in UTIs is extremely low. To the best of our knowledge, only six cases of 
pediatric UTIs caused by $S$. epidermidis have been reported [6-9].

Here, we report the case of an immunocompetent child receiving continuous antibiotic prophylaxis (CAP) with severe bilateral vesicoureteral reflux (VUR), who developed pyelonephritis caused by methicillin-resistant S. epidermidis despite having no urinary catheter.

\section{Case presentation}

An 18-month-old Japanese boy with rapid onset high fever for the previous 12 hours presented to our emergency room. His perinatal and family history was unremarkable. There was no record of previous immunological problems.

At 10 months of age, he was admitted to our hospital for examination owing to a fever of above $39^{\circ} \mathrm{C}$, lasting for two days, for an unknown reason. Laboratory examinations revealed leukocytosis (white blood cell (WBC) counts: $20,410 / \mu \mathrm{L}$ ), high absolute neutrophil counts (ANCs: $10,880 / \mu \mathrm{L}$ ), and high levels of C-reactive protein (CRP: $6.39 \mathrm{mg} / \mathrm{dL}$ ). Although his urine analysis did not reveal pyuria, contrast-enhanced computed tomography revealed that a part of his left renal parenchyma showed decreased contrast enhancement, indicating pyelonephritis, which was also observed in the right small kidney and compensatory hypertrophic left kidney (Figure 1). He was thus diagnosed with pyelonephritis, and empirically treated with intravenous ceftriaxone $\left(100 \mathrm{mg} / \mathrm{kg}^{-1} /\right.$ day $^{-1}$ at 24-hour intervals). Subsequently, a culture of urine obtained through catheterization revealed the presence of Enterococcus faecalis, which was susceptible to penicillins (Table 1). The urine was cultured, incubated on blood agar plates and bromothymol blue lactate agar plates at $37^{\circ} \mathrm{C}$ for species identification, and evaluated by slide culture for quantification of the organism. Moreover, we assessed the antimicrobial susceptibility of the isolate according to the Clinical and Laboratory Standards Institute criteria. The treatment was then changed to oral amoxicillin $\left(60 \mathrm{mg} / \mathrm{kg}^{-1} / \mathrm{day}^{-1}\right.$

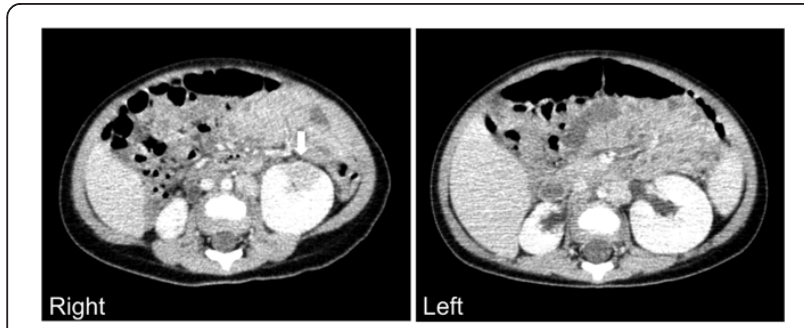

Figure 1 Enhanced computed tomography findings indicating left pyelonephritis. The right side image reveals decreased contrast enhancement in a part of the left renal parenchyma (arrow), indicating pyelonephritis. The left side image indicates the right small kidney and compensatory hypertrophic left kidney.
Table 1 Antibiotic susceptibility for each uropathogen

\begin{tabular}{lll}
\hline & $\begin{array}{l}\text { First } \\
\text { pyelonephritis } \\
\text { Enterococcus } \\
\text { faecalis }\end{array}$ & $\begin{array}{l}\text { Second } \\
\text { pyelonephritis } \\
\text { Staphylococcus } \\
\text { epidermidis }\end{array}$ \\
\hline $\begin{array}{l}\text { Benzylpenicillin } \\
\text { Oxacillin }\end{array}$ & $\mathrm{S}$ & $\mathrm{R}$ \\
Ampicillin & $\mathrm{R}$ \\
Sulbactam/ampicillin & $\mathrm{S}$ & $\mathrm{R}$ \\
Cefazolin & & $\mathrm{R}$ \\
Cefotiam & & $\mathrm{R}$ \\
Imipenem/cilastatin & & $\mathrm{R}$ \\
Gentamicin & & $\mathrm{S}$ \\
Amikacin & & $\mathrm{S}$ \\
Levofloxacin & $\mathrm{R}$ & $\mathrm{S}$ \\
Minocycline & $\mathrm{S}$ & $\mathrm{S}$ \\
Vancomycin & $\mathrm{S}$ & $\mathrm{S}$ \\
Linezolid & $\mathrm{S}$ & $\mathrm{S}$ \\
Trimethoprim- & $\mathrm{S}$ & $\mathrm{S}$ \\
sulfamethoxazole & $\mathrm{R}$ & \\
\hline S: suscepto R resstant & &
\end{tabular}

S: susceptible, R: resistant.

in three doses). He completed a three-week course of treatment and his symptoms were promptly resolved.

At 12 months of age, we performed a voiding cystourethrography, which showed severe bilateral VUR (right side grade V, left side grade III; Figure 2). Therefore, he was started on CAP with prophylactic-dose trimethoprim/sulfamethoxazole (TMP/SMX: 2mg/kg TMP and $10 \mathrm{mg} / \mathrm{kg}$ SMX per day in one single dose); he did not develop recurrent UTIs for the next six months.

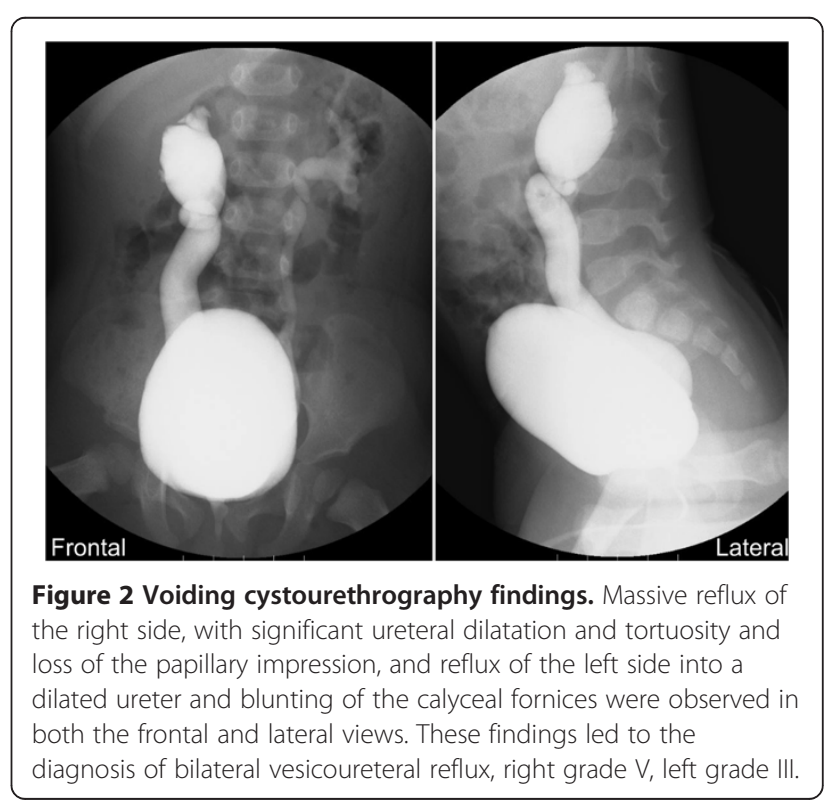


When he presented to our hospital again at 18 months of age, his body temperature was $39^{\circ} \mathrm{C}$. Other clinical examination findings were unremarkable. He had severe phimosis. The only abnormal laboratory findings were leukocytosis (WBC counts: 14,460/ $\mu \mathrm{L}$ ) and high ANCs $(10,450 / \mu \mathrm{L})$, but CRP levels were only slightly elevated $(1.81 \mathrm{mg} / \mathrm{dL})$. The urine analysis revealed five to nine WBCs per high-power field and a remarkably elevated level of $\beta 2$ microglobulin $(1,490 \mu \mathrm{g} / \mathrm{L})$. Gram staining of urine obtained through catheterization revealed grampositive cocci. While considering sample contamination, we also suspected pyelonephritis because there was no apparent source of the fever, and the urine sample was obtained by catheterization with a proper procedure. Moreover, during a routine examination two weeks prior, his urine analysis showed less than five WBCs per highpower field, despite the fact that it was bag-collected, and the $\beta 2$ microglobulin level was within the normal range $(195 \mu \mathrm{g} / \mathrm{L})$.

Since his clinical condition was generally good despite the high fever and he was not dehydrated, we determined that oral antibiotic treatment was possible. We planned to administer oral ampicillin after collecting blood and urine cultures, but we abandoned the treatment plan as he came to dislike the taste of oral amoxicillin subsequent to the prescription at first pyelonephritis at 10 months of age. Therefore, we selected tosufloxacin (oral fluoroquinolone, $12 \mathrm{mg} / \mathrm{kg}^{-1} /$ day $^{-1}$ in two doses). The fever promptly declined one day after antibiotic therapy was started. After two days, we performed repeated blood examinations, which showed elevated CRP and WBC levels $(12.2 \mathrm{mg} / \mathrm{dL}$ and $16,140 / \mu \mathrm{L}$, respectively), but significantly decreased ANCs levels $(6,843 / \mu \mathrm{L})$. In addition, we obtained a positive urine culture result indicating the presence of methicillinresistant S. epidermidis $\left(10^{7}\right.$ colony-forming units per milliliter) as single bacterial species; the blood culture was negative for any bacterial organisms. Thus, our final diagnosis was pyelonephritis caused by community-acquired methicillin-resistant S. epidermidis, and, based on the result of the antibiotic susceptibility test, the antibiotics were adjusted to 'therapeutic-dose' TMP/SMX (10 mg/kg TMP and $50 \mathrm{mg} / \mathrm{kg}$ SMX per day in two doses; Table 1). The total antibiotic treatment duration was two weeks, and his fever did not reoccur. Because he had severe bilateral VUR and developed recurrent pyelonephritis despite receiving CAP, antireflux surgery was performed at 20 months of age. He has not had a reoccurrence of pyelonephritis to date.

\section{Discussion}

S. epidermidis owes its pathogenic success to two major features: its natural niche on human skin, thus resulting in ready access to any device inserted or implanted across the skin, and its ability to adhere to biomaterials and form a biofilm [10,11]. The organisms adhere to a prosthetic material and then form multilayered clusters that become embedded in an exopolysaccharide matrix, thus forming a biofilm. The biofilm protects the organisms from phagocytic cells and reduces the penetration of antibiotics, and thus appears to facilitate infection by shielding these normally low-virulent organisms from elimination by host defenses or antimicrobial therapy $[3,10,11]$.

Treatment of S. epidermidis infections is generally difficult because of increasing resistance to many antibiotics. In particular, the number of strains showing resistance to methicillin, an antibiotic of first choice against staphylococci, has been increasing rapidly for many years [12]. Approximately 75 to $90 \%$ of hospital isolates of S. epidermidis show resistance to methicillin worldwide [13]. In addition to methicillin resistance, most $S$. epidermidis isolates are resistant to other antibiotics; most strains were found to be resistant to fluoroquinolones and macrolides, and many strains were resistant to clindamycin and TMP/SMX in North America and the United Kingdom [10]. Although this is mostly due to the high antibiotic resistance rates among nosocomial S. epidermidis isolates, treatment failure is also associated with the ability of $S$. epidermidis to form biofilms on inert surfaces of medical devices, increasing the difficulty of removal of these sticky, multilayered aggregates of bacteria [14].

In pediatric UTIs, S. epidermidis is rarely isolated; indeed, in a review of the English literature, we found only six reported cases (Table 2) [6-9]. In contrast to our case, all six reported cases first developed pyelonephritis caused by $S$. epidermidis, and no precedent antibiotics had been administered. None of the cases, including our case, involved a urinary catheter, and all were immunocompetent. In addition, all but one of the patients were preadolescents. It is noteworthy that all cases had urinary abnormalities; four were severe VUR (Grade III and above), and although the other two did not have reflux, they did have had bladder diverticulum, which can lead to urine stasis. These previous reports and the details of our case demonstrate that a UTI caused by S. epidermidis can occur in individuals of all ages, even in immunocompetent children, and all susceptible patients have apparent underlying urinary tract abnormalities. Although no detailed description of the susceptibility pattern was provided and methicillin-resistant S. epidermidis was not specified in previous reports, antibiotics other than penicillins and cephalosporins are considered necessary in such cases.

The pathogenesis of S. epidermidis UTIs in previously reported cases and our case remains unclear. Numerous studies have clearly indicated that the ability to form biofilms on inert surfaces represents a typical feature of 
Table 2 Literature review of pediatric cases of urinary tract infections caused by Staphylococcus epidermidis

\begin{tabular}{|c|c|c|c|c|c|}
\hline Source & Age (years) & uWBCs (/hpf) & Sensitive & Resistance & Urinary abnormalities \\
\hline \multirow[t]{2}{*}{ Hagler et al. 1990 [6] } & 9 & $>100$ & $\begin{array}{l}\text { Cephalosporins, VCM, TMP, } \\
\text { EM, nitrofurantoin }\end{array}$ & $\mathrm{ABPC}$ & No VUR, Bladder diverticulum \\
\hline & 11 & $20-30$ & EM, nitrofurantoin, tetracycline & $\mathrm{ABPC}$ & No VUR, Bladder diverticulum \\
\hline \multirow[t]{2}{*}{ Hall et al.1994 [7] } & 6 & None & VCM, TMP/SMX, clindamycin & ABPC, cephalosporins & VUR: Bilateral Gr III, Duplications \\
\hline & 7 & $3-5$ & Cephalosporins & - & $\begin{array}{l}\text { VUR: Right Gr III, Left Gr II } \\
\text { Posterior urethral valve }\end{array}$ \\
\hline $\begin{array}{l}\text { McDonald et al. } \\
1994[8]\end{array}$ & 11 & $5-10$ & - & - & VUR: Right Gr III \\
\hline $\begin{array}{l}\text { Upadhyayula et al. } \\
2012 \text { [9] }\end{array}$ & 0.6 & $5-10$ & VCM, TMP/SMX, GM & Ceftriaxone & VUR: Right Gr V, Left Gr IV \\
\hline Present & 1.5 & $5-9$ & $\begin{array}{l}\text { VCM, TMP/SMX, GM, amikacin, } \\
\text { minocycline, levofloxacin }\end{array}$ & Penicillins, cephalosporins & $\begin{array}{l}\text { VUR: Right Gr V, Left Gr III } \\
\text { Right small kidney }\end{array}$ \\
\hline
\end{tabular}

ABPC: ampicillin; EM: erythromycin; GM: gentamycin; hpf: high power field; Gr: grade; TMP/SMX: trimethoprim/sulfamethoxazole; uWBCs: urine white blood cells; VCM: vancomycin; VUR: vesicoureteral reflux.

nosocomial infections. Bacteria adhere to a surface by unspecific factors, such as hydrophobicity and surface charge, and the initial adherence stage is followed by accumulation of the biofilm $[11,12]$. As mentioned above, our case did not have a urinary catheter at presentation and no evidence of an immunological problem. Therefore, based on previous reports, severe VUR associated with dilated ureters may somehow be a predisposing risk factor for S. epidermidis infection. Moreover, it is noteworthy that in our case, S. epidermidis was susceptible to TMP/ SMX, even though our patient had previously received CAP with prophylactic-dose TMP/SMX. Our patient had already experienced pyelonephritis caused by enterococci, which was resistant to TMP/SMX; however, we selected $\mathrm{TMP} / \mathrm{SMX}$ as the antimicrobial prophylaxis to prevent UTIs caused not by enterococci, but by gram-negative rods, which are primary uropathogens even in recurrent UTIs or severe VUR. According to these conditions, we speculated that the presence of bilateral dilated ureters causing persistent urinary stasis allowed for S. epidermidis to produce a protective biofilm and enhanced its adhesion to the mucosal surface of the ureters. In addition, the most likely explanation for why our patient developed pyelonephritis caused by $S$. epidermidis susceptible to TMP/ SMX regardless of receiving CAP with TMP/SMX is as follows. Normally, the simple and compound papillae in the kidney have an antireflux mechanism that prevents urine in the renal pelvis from entering the collecting tubules. However, some VURs result in intrarenal reflux, and subsequently, the infected urine stimulates an immunologic and inflammatory response in the kidney parenchyma, causing pyelonephritis [15]. In addition, as $S$. epidermidis is surrounded by a biofilm, it can resist phagocytosis, and further impair the penetration of many antibiotics. Consequently, although the organisms were susceptible to TMP/SMX in vitro, the prophylactic effect might become weak in vivo.
In reviewing the clinical course of our case report retrospectively, we have highlighted the main factors contributing to the potential for underdiagnoses or misdiagnoses of S. epidermidis pyelonephritis. Although our patient did not show any symptoms other than high fever and urine analysis indicated pyuria by definition, the nitrate test results were negative and the CRP level as the serum inflammatory marker was only slightly elevated. Therefore, we would not have suspected pyelonephritis at initial presentation from these examination results alone. However, Gram staining of urine samples obtained aseptically through catheterization showed significantly positive gram-positive cocci, which indicated the possibility of pyelonephritis. Thus, we empirically decided to administer antibiotics. Two days later, his CRP levels were markedly elevated to $12.2 \mathrm{mg} / \mathrm{dL}$, despite the fact that his fever was resolved, and ANCs decreased. Ultimately, a single $S$. epidermidis identified from the urine specimen obtained aseptically through catheterization was found to grow at a rate of $10^{7}$ colony-forming units per milliliter. On the basis of these findings and the antimicrobial treatment response, we confirmed that S. epidermidis was the uropathogen. Fortunately, we could select the antibiotics appropriate for methicillin-resistant S. epidermidis. If we had initially considered that the presence of $S$. epidermidis was due to sample contamination, appropriate therapy, including suitable antibiotics for a sufficient duration and the subsequent surgery, would not have been provided. Furthermore, inappropriate or insufficient antibiotic treatment might have caused urosepsis or renal damage. Our case indicates that in ambulatory practice, opportunistic pathogens like S. epidermidis have the potential to be underdiagnosed or misdiagnosed, thereby increasing the risk of treatment failure.

With regard to laboratory findings of pyelonephritis, leukocytosis, neutrophilia and elevated CRP level are common in the acute phase of pyelonephritis [15]. 
Table 3 The levels of C-reactive protein (CRP) at the time of diagnosis, sensitivity, specificity, positive (PPV) and negative predictive values (NPV) of CRP for diagnosis of acute pyelonephritis in children (cut-off values of $\mathbf{2 m g} / \mathrm{dl}$ )

\begin{tabular}{lllllll}
\hline Reference number & $\mathbf{N}$ & Mean \pm SD, $\mathbf{~ m g} / \mathbf{d l}$ & Sensitivity, $\%$ & Specificity, \% & PPV, \% & NPV, \% \\
\hline Smolkin et al. 2002 [16] & 42 & 12 & 100 & 18.5 & 30.9 & 100 \\
Pecile et al. 2004 [17] & 53 & $10.6 \pm 2.6$ & 94.4 & 31.9 & 61.4 & 83.3 \\
Nikfar et al. 2010 [18] & 62 & ND & 80 & 65 & 79 & 67 \\
Xu et al. 2014 [19] & 21 & $6.82 \pm 3.94$ & 85.71 & 48 & 50 & 80 \\
\hline
\end{tabular}

ND: not described.

However, these are nonspecific markers of bacterial infection, and their elevated levels do not prove acute pyelonephritis [15]. Although our patient first revealed leukocytosis and neutrophilia at the second pyelonephritis, his CRP level was not significantly elevated $(1.81 \mathrm{mg} / \mathrm{dl})$. However, two days after presentation, his CRP level was remarkably elevated $(12.2 \mathrm{mg} / \mathrm{dl})$, while the clinical symptoms improved. Some clinical studies have examined the significance of laboratory data, including CRP, to diagnose acute pyelonephritis in children (Table 3) [16-19]. These studies indicated that CRP levels have a wide range and are not always elevated, even in acute pyelonephritis. In addition, it was shown that although the specificity of CRP was low, the sensitivity was relativity high. Also, these studies had important limitations. Despite it being well known that CRP level is affected by the timing of examination, the time of blood examinations from the onset was not described. Thus, as seen in our patient, low CRP levels (especially lower than $2 \mathrm{mg} / \mathrm{dl}$ ) could not exclude acute pyelonephritis, and it was considered that CRP was only one of the 'predictive' but poor 'diagnostic' markers of pyelonephritis.

Yet, it remains a difficult challenge to differentiate true $S$. epidermidis infection from contaminants. This is because S. epidermidis occupy a prominent position in the commensal flora of the human skin and mucous membranes and are thus frequently encountered as culture contaminants. Regarding bacteremia, approximately 1 to $6 \%$ of blood cultures are contaminated, and coagulasenegative staphylococci (usually S. epidermidis) are responsible for between 70 and $80 \%$ of such cases [10]. However, in UTIs, the epidemiology of contaminants is not apparent. Therefore, it is important for clinicians to be aware of the possibility of 'true' S. epidermidis infection when it is identified in urine culture, even in immunocompetent cases or in cases without indwelling medical devices, and not simply presume that the bacteria represent contamination. A variety of clinical and laboratory parameters, including urine collection techniques, images of urinary tracts, and the subsequent response of antibiotic treatment should be examined and evaluated in such cases. Based on the clinical course of our case and the literature review, we suggest that urinary tract abnormalities are a risk factor for S. epidermidis infection in pediatric UTIs. Further studies are needed to identify the prevalence of S. epidermidis UTIs in children and the factors responsible for developing the UTI.

\section{Conclusions}

Our case raises three important considerations. First, even immunocompetent children without a urinary catheter can develop pyelonephritis caused by methicillin-resistant S. epidermidis. Second, because S. epidermidis is one of the most commonly diagnosed contaminants in various clinical settings, it can easily be underdiagnosed or misdiagnosed in pediatric UTIs if an accurate urine culture is not obtained using catheterization or suprapubic aspiration. Third, clinicians should consider not only enterococci but also staphylococci as potential uropathogens when Gram staining of appropriately obtained urine reveals the presence of gram-positive cocci, particularly in children with urinary abnormalities and/or CAP.

\section{Consent}

Written informed consent was obtained from the patient's legal guardian for publication of this case report and accompanying images. A copy of the written consent is available for review by the Editor-in-Chief of this journal.

\section{Abbreviations}

S. epidermidis: Staphylococcus epidermidis; UTIs: urinary tract infections;

CAP: continuous antibiotic prophylaxis; VUR: vesicoureteral reflux; WBC: white blood cell; ANCs: absolute neutrophil counts; CRP: C-reactive protein; TMP/ SMX: trimethoprim/sulfamethoxazole.

Competing interests

The authors declare that they have no competing interests.

\section{Authors' contributions}

$\mathrm{HK}$ is the first author and major contributor in writing the manuscript. HS and $Y T$ collected and interpreted the patient data. All authors read and approved the final manuscript.

Acknowledgments

We appreciate all technicians of the laboratory of Suwa Central Hospital for providing the blood and microbiology data.

Received: 16 May 2014 Accepted: 30 October 2014

Published: 9 December 2014 


\section{References}

1. Todd JK: Coagulase-negative staphylococci. In Nelson Textbook of Pediatrics. 19th edition. Edited by Kliegman RM, Stanton BF, St Geme JW III Schor NF, Behrman RE. Philadelphia: Saunders; 2011:909-910.

2. American Academy of Pediatrics: Staphylococcal infections. In Red Book: 2012 Report of the Committee on Infectious Diseases. 29th edition. Edited by Pickering LK, Baker CJ, Kimberlin DW, Long SS. Elk Grove Village, IL: American Academy of Pediatrics; 2012:653-668.

3. Moffett SE, Frazee BW, Stein JC, Navab B, Maselli J, Takhar SS, Gonzales R: Antimicrobial resistance in uncomplicated urinary tract infections in 3 California EDs. Am J Emerg Med 2012, 30:942-949.

4. Lo DS, Shieh HH, Ragazzi SL, Koch VH, Martinez MB, Gilio AE: Communityacquired urinary tract infection: age and gender-dependent etiology. J Bras Nefro 2013, 35:93-98.

5. Abdullah FE, Memon AA, Bandukda MY, Jamil M: Increasing ciprofloxacin resistance of isolates from infected urines of a cross-section of patients in Karachi. BMC Res Notes 2012, 27:696.

6. Hagler S, Dobkin D: Urinary tract infection in the male caused by Staphylococcus epidermidis associated with diverticulum of the bladder. Clin Pediatr (Phila) 1990, 29:527-528.

7. Hall DE, Snitzer JA 3rd: Staphylococcus epidermidis as a cause of urinary tract infections in children. J Pediatr 1994, 124:437-438.

8. McDonald JA, Lohr JA: Staphylococcus epidermidis pyelonephritis in a previously healthy child. Pediatr Infect Dis J 1994, 13:1155-1156.

9. Upadhyayula S, Kambalapalli M, Asmar BI: Staphylococcus epidermidis urinary tract infection in an infant. Case Rep Infect Dis 2012, 2012:983153.

10. Rupp ME: Clinical characteristics of infections in humans due to Staphylococcus epidermidis. Methods Mol Biol 2014, 1106:1-16.

11. von Eiff C, Peters $G$, Heilmann C: Pathogenesis of infections due to coagulase-negative staphylococci. Lancet Infect Dis 2002, 2:677-685.

12. Otto M: Staphylococcus epidermidis-the 'accidental' pathogen. Nat Rev Microbiol 2009, 7:555-567.

13. Chovanová R, Mikulášová M, Vaverková S: In vitro antibacterial and antibiotic resistance modifying effect of bioactive plant extracts on methicillin-resistant Staphylococcus epidermidis. Int J Microbiol 2013, 2013:760969.

14. Mack D, Rohde H, Harris LG, Davies AP, Horstkotte MA, Knobloch JK: Biofilm formation in medical device-related infection. Int J Artif Organs 2006, 29:343-359.

15. Elder JS: Urinary tract infections. In Nelson Textbook of Pediatrics. 19th edition. Edited by Kliegman RM, Stanton BF, St Geme JW III, Schor NF, Behrman RE. Philadelphia: Saunders; 2011:1829-1834.

16. Smolkin V, Koren A, Raz R, Colodner R, Sakran W, Halevy R: Procalcitonin as a marker of acute pyelonephritis in infants and children. Pediatr Nephrol 2002, 17:409-412.

17. Pecile P, Miorin E, Romanello C, Falleti E, Valent F, Giacomuzzi F, Tenore A: Procalcitonin: a marker of severity of acute pyelonephritis among children. Pediatrics 2004, 114:249-254.

18. Nikfar R, Khotaee G, Ataee N, Shams S: Usefulness of procalcitonin rapid test for the diagnosis of acute pyelonephritis in children in the emergency department. Pediatr Int 2010, 52:196-198.

19. Xu RY, Liu HW, Liu JL, Dong JH: Procalcitonin and C-reactive protein in urinary tract infection diagnosis. BMC Urol 2014, 14:45.

\section{Submit your next manuscript to BioMed Central and take full advantage of:}

- Convenient online submission

- Thorough peer review

- No space constraints or color figure charges

- Immediate publication on acceptance

- Inclusion in PubMed, CAS, Scopus and Google Scholar

- Research which is freely available for redistribution

Submit your manuscript at www.biomedcentral.com/submit
Ciomed Central 